\title{
INTEGRATION OF SIMULATION WITH ENTERPRISE MODELS
}

\author{
K. Srinivasan \\ Distributed Enterprise Architecture Laboratory \\ Intel Corporation \\ Chandler, Arizona 85226, U.S.A.
}

\author{
Sundaresan Jayaraman \\ School of Textile \& Fiber Engineering \\ Textile Information Systems Research Laboratory \\ Georgia Institute of Technology \\ Atlanta, GA 30332-0295, U.S.A.
}

\begin{abstract}
The Enterprise Modeling Framework (EMF) consists of a methodology for modeling the three major facets of an enterprise, viz., function, information and dynamics. Its main goal is to enable integration of enterprise through shared, consistent models. EMF activity (function) and entity (information) models have been designed to include the information needed for discrete-event simulation of the enterprise functions. The EMF-SIMAN model generator automatically generates SIMAN code from these models. This tool has been applied to solve a real-world problem whose solution required the integration of enterprise modeling with discrete-event simulation. This paper discusses how EMF integrates simulation with enterprise modeling, the resulting benefits, and a real-world application of EMF.
\end{abstract}

\section{INTRODUCTION}

Simulation of an enterprise (e.g., an apparel manufacturing company) requires a detailed understanding of the operations in the enterprise including the process sequences, the resources (machines, humans and materials) and the constraints governing the processes and the resources. Most of this information is contained in the underlying function and information models of the enterprise. The process sequences are often complex and the number of resources employed by an enterprise is large. Moreover, the process sequence and the information on the resources change over time. Replicating this information in a simulation model not only imposes an unnecessary burden on the developers of the model but can also lead to inconsistency. Enterprise Modeling Framework (EMF) addresses this problem through (i) a modeling methodology that allows the integration of the function, information and dynamics models without any redundancy; (ii) tools for developing these models in a seamless fashion; and (iii) automatic generation of SIMAN code for simulation.

\section{AN OVERVIEW OF ENTERPRISE MODELING FRAMEWORK}

The design of an integrated information system for an enterprise requires a complete understanding of the various functions involved in the working of an enterprise, their information needs and dynamics. An understanding of these aspects of an enterprise is promoted by formal models of an enterprise. EMF consists of a methodology for modeling the three major facets of an enterprise, viz., function, information and dynamics (Jayaraman 1989) and software tools implementing the methodology. These facets of an enterprise have been broadly structured using the objectoriented paradigm into three EMF models, viz., The Activity Model, the Entity Model and the Knowledge Model (Srinivasan and Jayaraman 1991). The EMF Activity model is a representation of the various functions performed in operating an enterprise and is based on the $\mathrm{IDEF}_{0}$ Methodology (ICAM 1981). The EMF Entity model represents entities such as operators, equipment, orders and design using an object-oriented model. The Knowledge model comprises the knowledge and heuristics employed for carrying out different functions in a manufacturing enterprise. This knowledge can be maintained in the form of separate knowledge bases or can be integrated with the EMF models in the form of constraints and triggers associated with various entities and their attributes. EMF is implemented in Common LISP (Srinivasan 1993).

\section{APPLICATION OF EMF}

Terry Manufacturing, an apparel manufacturing company in Roanoke, Alabama, produces battle-dress uniform (BDU) or camouflage coats for the US 
Department of Defense (DoD) and uniforms for restaurant chains in a unique "shared production" partnership. The company needed the capability to ramp $u p$ its production for $\mathrm{DoD}$ in the event of a mobilization by redeploying its machinery and personnel rapidly from other product lines. The following were necessary to achieve this goal:

- Comprehensive understanding of the differences in the processes and resource needs for different products (BDU Coats, Restaurant Uniforms, etc.);

- Integration of the sales, planning, manufacturing, payroll and other information systems through a shared database; and

- Ability to answer questions such as which resource will be the bottleneck if supply to DoD has to be ramped up by say $25 \%$, and how much ramp-up can be achieved with available resources.

A two-pronged approach was adopted to carry out this effort in collaboration with Terry Manufacturing personnel. First, a comprehensive enterprise model encompassing the function and information facets was developed and used as the framework for the design and development of a shared database. Second, with the enterprise model as the basis, discrete-event simulation models were created and used to determine the resources needed for ramping up the supply to DoD by various levels from $10 \%$ to $50 \%$ and also to identify the bottlenecks.

While the models developed for Terry Manufacturing are excellent examples demonstrating the need for integration between enterprise function, information and dynamics models, they are too complex for description in this paper (the process for manufacturing the BDU Coats involves more than 60 operations). Hence, a simple hypothetical example is used in the next section to describe the EMF-SIMAN generator implementation. Srinivasan,et al. (Srinivasan, Terry and Jayaraman 1993) describe the models developed for Terry Manufacturing and the benefits in detail.

\section{IMPLEMENTATION OF EMF AND EMF- SIMAN GENERATOR}

EMF provides different modeling constructs to reflect the differences between flow-shops and job-shops. A flow-shop usually produces a limited number of products and new products are not introduced frequently. In EMF, the process plan for flow-shops is explicitly modeled as a part of the Activity Model. Figure 1 shows the processes involved in the manufacturing of a cylindrical container in a flow-shop using extended IDEF $_{0}$ notation. On the other hand, a job-shop produces several products at a time. Also, new one-time-only orders for products are common. To accommodate such frequent changes in products and process plans, the process sequence is attached to the entity representing the product.

\subsection{Simulation of Flow-shops}

In the simplified Activity Model of a flow-shop (Figure 1) only one product, viz., a cylindrical container, is manufactured from sheet metal. The figure shows the decomposition of the Make Cylindrical Container activity. The static aspects of the functions include the interface to the function in terms of its Inputs, Controls, Outputs and Mechanisms. For example, consider the activity Cut Metal in Figure 1: its Input is Sheet Metal, Control is Process Parameters, Outputs are Cut Partl and CutPart2, and the Mechanisms are the Cutting Machine and Cutting Operator. In EMF, Mechanisms such as Cutting Machine and Cutting Operator are modeled as sub-classes of a built-in generic class Resource and inherit its attribute Total Number. The number of a particular resource available is specified using this attribute. The dynamic aspects of an activity include the following: (i) the size of the lot processed at a time (its default value is one, i.e., parts are processed one at a time unless specified otherwise); (ii) the distribution followed by the process time (triangular, uniform, exponential and normal are the distributions currently supported by EMF); (iii) the relative proportions of the different types of parts (outputs) produced. Figure 2 shows the EMF editors used to specify these aspects for the activity Cut Metal in the present example.

\subsubsection{Determining Assembly Processes}

In Figure 1, the activity Assemble Container has two inputs -- one is a queue of Cylindrical Parts and the other is a queue of Bottom Pieces. The Assemble Container activity can be triggered only if entities are present in both the input queues. On the other hand, certain activities may receive inputs through multiple queues, but can be triggered by the presence of an entity in any one of the input queues. The latter is the default in EMF. It waits for entities in multiple input queues based on the following two criteria:

- An activity is defined to be of the type Assembly; and

- The input entity types are components of the output entity type. 


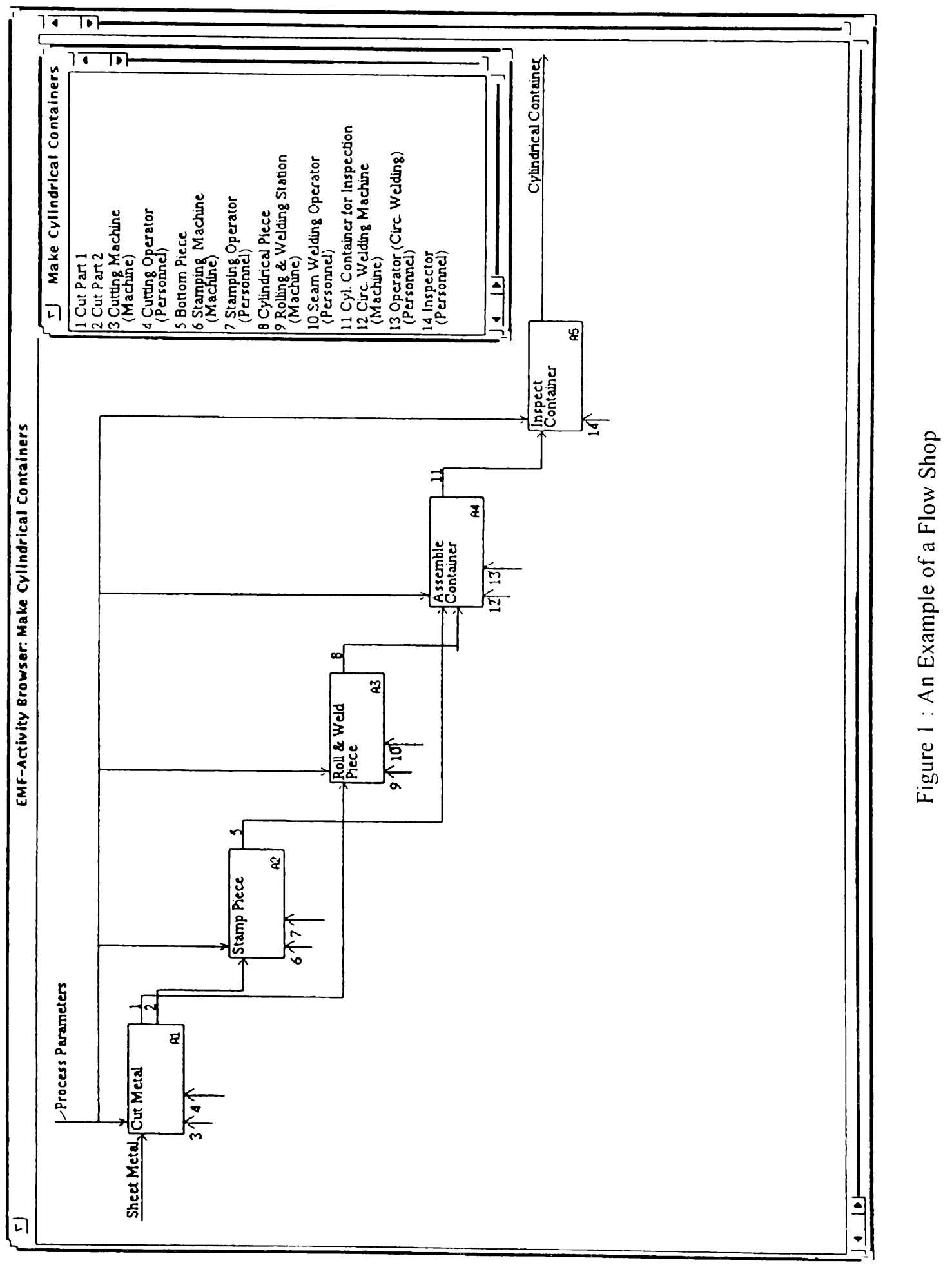




\subsubsection{Specifying Branches in Material Flow}

It can be seen from Figure 1 that the Cut Metal activity supplies both the Stamp Piece and Roll \& Weld activities with their inputs. When EMF encounters an activity feeding multiple activities, it prompts the user for the proportion of parts to be fed to each process through the Event Editor (Figures 2a and 2b). In the current case, since each container is made from one Bottom Piece and one Cylindrical Part, a proportion of $50 \%$ is assigned for each output queue.

\begin{tabular}{|c|c|c|}
\hline$\Xi$ & Event Editor & \\
\hline Save Event Load Event & Next Event) & \\
\hline \multicolumn{3}{|l|}{ Activity: cut-metal } \\
\hline \multicolumn{3}{|l|}{ Event: cut } \\
\hline \multicolumn{3}{|l|}{ Lot Size: 1} \\
\hline \multicolumn{3}{|c|}{ Process Time Distribution: uniform } \\
\hline \multicolumn{3}{|c|}{ Percentages of Outputs } \\
\hline Output & & Percentage \\
\hline Cut Part1 & $:$ & - \\
\hline Cut Part2 & $:$ & $-1=$ \\
\hline
\end{tabular}

Figure 2a: User Interface for Defining a Process Step (Event Cutl of the Activity Cut Metal)

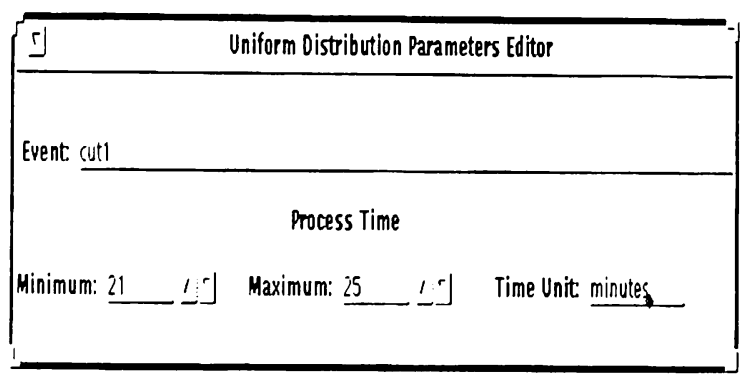

Figure 2b: User Interface for Defining a Process Step Specifying Distribution Parameters for the Event Cutl

\subsubsection{Specifying Simulation Parameters and Generating Simulation Code}

In addition to describing all activities in terms of their static and dynamic features, it is necessary to specify parameters for running the simulation experiments (Figure 3). The parameters to be specified are (i) the scope of the simulation model; (ii) the total simulation run time; (iii) the time to be allowed for the system to reach steady state; and (iv) the number of simulation runs. After the modeler fills in all the simulation experiment parameters, the model and experiment files required for a SIMAN simulation model (Pegden, Shannon and Sadowski 1990) are generated by EMF.

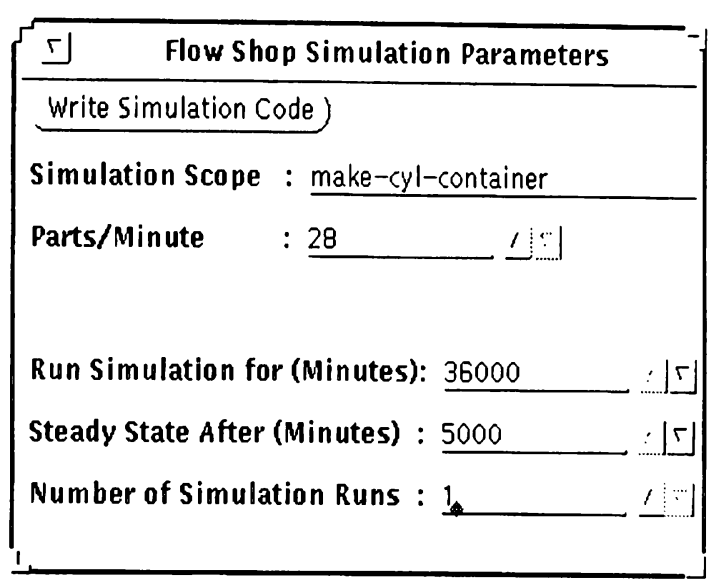

Figure 3: Parameters for a Flow-shop Simulation

\subsection{Simulation of Job-shops}

As mentioned before, the major difference between jobshop models and flow-shop models in EMF is that the process plans are encapsulated with the product type in a flow-shop model. This minimizes changes needed to the models when new product types are introduced.

While specifying the simulation experiment for a jobshop, the form (Figure 4) displays a list of all sub-classes (Part1, Part2 and Part3) of the built-in class Material for Manufacturing, and lets the user specify what proportion of the arriving parts are of the particular class; if the user wishes to exclude a particular class from the simulation experiment, its percentage can be set to zero. Apart from the proportion of different part types, the parameters to be specified are the part arrival rate, total simulation run time, time taken for the system to reach steady state and the number of simulation runs.

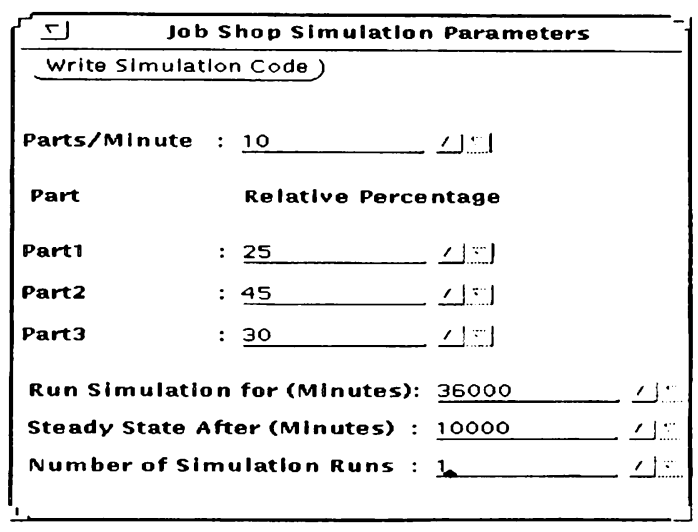

Figure 4: Parameters for a Job-shop Simulation 


\section{CONCLUSIONS}

EMF facilitates enterprise integration through shared models of the function, information and dynamics facets of an enterprise. EMF-SIMAN model generator automatically generates SIMAN models by extracting the required information from EMF models. This approach offers two important benefits, viz., reducing the load on the developers of the simulation models and ensuring consistency and integrity between the enterprise function and information models used in the operation of the enterprise and the simulation models used for planning and decision-making. The experience in using EMF with Terry Manufacturing to plan for rapid redeployment of resources validates the usefulness of this approach.

\section{ACKNOWLEDGMENTS}

The authors wish to thank the National Science Foundation for funding this research in part through Presidential Young Investigator Research Award N0. DDM-8957861, the U.S. Defense Logistics Agency for research funding under DLA-900-87-D-0018/0001 and personnel from Terry Manufacturing for their participation.

\section{REFERENCES}

Integrated Computer-Aided Manufacturing (ICAM). 1981. Function Modeling Manual IDEFO, Materials Laboratory, Air Force Wright Aeronautical Laboratories, AFSC, Wright-Patterson AFB, OH.

Jayaraman, S. 1989. On a Manufacturing Enterprise Architecture, IJCAI 1989, Workshop on Integrated Architectures for Manufacturing, August, Detroit, MI.

Pegden, C.D., R.E. Shannon, and R. Sadowski. 1990. Introduction to Simulation Using SIMAN, McGrawHill, Inc., New York, NY.

Srinivasan, K., and S. Jayaraman. 1991. MEA: An Object-Oriented Modeling Framework for Modeling a Manufacturing Enterprise, Workshop on ObjectOriented Programming in AI, AAAI-9I, July, Anaheim, CA.

Srinivasan, K. 1993. Design and Development of an Enterprise Modeling Framework, Doctoral Dissertation, Georgia Institute of Technology, Atlanta, Georgia, December 1993.

Srinivasan, K., R. Terry, and S. Jayaraman. 1993. An Information System for Shared Production: Design, Development \& Implementation, Proceedings Of the Twentieth International Apparel Research Conference, December, Atlanta, GA.

\section{AUTHOR BIOGRAPHIES}

K. SRINIVASAN is currently employed in the Distributed Enterprise Architecture Laboratory of Intel Corporation. He is involved in developing a strategy for application of distributed objects and components software technologies for inter- and intra-enterprise integration. $\mathrm{He}$ completed his $\mathrm{PhD}$ from Georgia Institute of Technology in December 1993. His research work focused on the development of the Enterprise Modeling Framework, an object-oriented methodology for modeling the function, information and dynamics facets of an enterprise. He obtained his M.S. in Textile Engineering from Georgia Institute of Technology in April 1990. His Masters thesis work was on developing a knowledge-based system for analyzing defects in woven fabrics. He also has a B.Tech. in Textile Engineering from Anna University, India.

SUNDARESAN JAYARAMAN is Professor of Textile Engineering at the Georgia Institute of Technology in Atlanta, GA. His current research interests are in the areas of enterprise modeling, computer-integrated manufacturing (CIM), knowledge-based systems (KBS) for textiles/apparel, and engineering design of textile structures including three-dimensional woven composites. Dr. Jayaraman received his Ph.D. degree from North Carolina State University, Raleigh, NC, and the M.Tech and B.Tech degrees from the University of Madras, India. Dr. Jayaraman is a recipient of the 1989 Presidential Young Investigator Award from the National Science Foundation for his research in the area of computer-aided manufacturing. In addition to other publications and presentations, Dr. Jayaraman is the author of a textbook from McGraw-Hill and has coauthored a book in the past for Osborne/McGraw-Hill. He was elected a Fellow of the Textile Institute (UK) in September 1994. He is a Technical Editor -- Information Technology -- for America's Textiles International, a leading textile trade publication. 